\title{
ON THE DERIVATIVE OF A POLYNOMIAL
}

\author{
N. K. GOVIL
}

ABSTRACT. If $p(z)=\sum_{v=0}^{n} a_{v} z^{v}$ is a polynomial of degree $n$ having all its zeros in $|z| \leqq K \leqq 1$, then it is known that $\max _{|z|=1}\left|p^{\prime}(z)\right| \geqq$ $(n /(1+K)) \max _{|z|=1}|p(z)|$. In this paper we consider the case when $K>1$ and obtain a sharp result.

1. The following result is due to Turán [4].

THEOREM A. If $p(z)$ is a polynomial of degree $n$ having all its zeros in $|z| \leqq 1$ then

$$
\max _{|z|=1}\left|p^{\prime}(z)\right| \geqq \frac{n}{2} \max _{|z|=1}|p(z)| .
$$

The result is sharp and equality holds in (1) if all the zeros of $p(z)$ lie on $|z|=1$.

More generally if $p(z)=a_{n} \prod_{v=1}^{n}\left(z-z_{v}\right)$ is a polynomial of degree $n$ having all its zeros in $|z| \leqq K \leqq 1$, then

i.e.

$$
\left|\frac{p^{\prime}\left(e^{i \theta}\right)}{p\left(e^{i \theta}\right)}\right| \geqq \operatorname{Re}\left(e^{i \theta} \frac{p^{\prime}\left(e^{i \theta}\right)}{p\left(e^{i \theta}\right)}\right)=\sum_{v=1}^{n} \operatorname{Re}\left(\frac{e^{i \theta}}{e^{i \theta}-z_{v}}\right) \geqq \sum_{v=1}^{n} \frac{1}{1+K}
$$

$$
\left|p^{\prime}\left(e^{i \theta}\right)\right| \geqq(n /(1+K))\left|p\left(e^{i \theta}\right)\right|, \quad \theta \text { real. }
$$

Choosing $\theta$ such that $\left|p\left(e^{i \theta}\right)\right|=\max _{|z|=1}|p(z)|$, we get

$$
\max _{|z|=1}\left|p^{\prime}(z)\right| \geqq \frac{n}{1+K} \max _{|z|=1}|p(z)| \text {. }
$$

In (2) equality holds for the polynomial $p(z)=((z+K) /(1+K))^{n}$.

The above argument does not hold for $K>1$ for then $\operatorname{Re}\left(e^{i \theta} /\left(e^{i \theta}-z_{v}\right)\right)$ may not be $\geqq 1 /(1+K)$.

Another proof of (2) is given in [2] where it is deduced by applying the following result (for another proof see [1, Theorem C, p. 503]) to the polynomial $z^{n} p(1 / z)$.

Received by the editors February 26, 1973.

AMS (MOS) subject classifications (1970). Primary 30A40; Secondary 30A04, $30 \mathrm{~A} 06$.

Key words and phrases. Inequalities in the complex domain, polynomials, extremal problems.

(c) American Mathematical Society 1973 
THEOREM B. If $p(z)$ is a polynomial of degree $n$, with $|p(z)| \leqq 1$ on $|z| \leqq 1$ and $p(z)$ has no zero in the disk $|z|<K, K \geqq 1$, then for $|z| \leqq 1$,

$$
\left|p^{\prime}(z)\right| \leqq n /(1+K) \text {. }
$$

The result is best possible and equality in (3) holds for $p(z)=((z+K) /(1+K))^{n}$.

Thus again the question as to what happens to (2) if $K>1$ remains unanswered. We settle the case $K>1$ by proving the following theorem.

THEOREM. If $p(z)=\sum_{v=0}^{n} a_{v} z^{v}$ is a polynomial of degree $n$ with $\max _{|z|=1}|p(z)|=1$ and $p(z)$ has all its zeros in the disk $|z| \leqq K, K \geqq 1$, then

$$
\max _{|z|=1}\left|p^{\prime}(z)\right| \geqq \frac{n}{1+K^{n}} .
$$

The result is best possible with equality for the polynomial $p(z)=$ $\left(z^{n}+K^{n}\right) /\left(1+K^{n}\right)$.

For $K>1$ the extremal polynomial turns out to be of the form $\left(z^{n}+K^{n}\right) /\left(1+K^{n}\right)$ whereas for $K<1$ it has the form $((z+K) /(1+K))^{n}$. Thus 1 is a critical value of $K$ for the problem under consideration and one should not expect the same kind of reasoning to work both for $K<1$ and for $K>1$.

2. For the proof of the theorem, we need the following lemmas.

LEMMA 1. If $p(z)=\sum_{v=0}^{n} a_{v} z^{v}$ is a polynomial of degree $n$ having all its zeros in the disk $|z| \leqq K, K \geqq 1$ then for $0 \leqq \theta<2 \pi,\left|p^{\prime}\left(K^{2} e^{i \theta}\right)\right| \geqq K^{n-2}\left|q^{\prime}\left(e^{i \theta}\right)\right|$, when $q(z)=z^{n}(p(1 / \bar{z}))$.

Proof of Lemma 1. The polynomial $P_{1}(z) \equiv p(K z)$ has all its zeros in the unit disk $|z| \leqq 1$ and so the polynomial $Q_{1}(z)=z^{n}\left(P_{1}(1 / \bar{z})\right)^{-} \equiv$ $z^{n}(p(K / \bar{z}))^{-} \equiv K^{n} q(z \mid K)$ has all its zeros in $|z| \geqq 1$. Since $\left|P_{1}(z)\right|=\left|Q_{1}(z)\right|$ on $|z|=1$, it follows that $\left|Q_{1}(z)\right| \leqq\left|P_{1}(z)\right|$ for $|z| \geqq 1$. Hence $Q_{1}(z)-\lambda P_{1}(z)$ has all its zeros in $|z|<1$ if $|\lambda|>1$. It then follows by the Gauss-Lucas theorem that all the zeros of the polynomial $Q_{1}^{\prime}(z)-\lambda P_{1}^{\prime}(z)$ also lie in $|z|<1$, which implies that $\left|Q_{1}^{\prime}(z)\right| \leqq\left|P_{1}^{\prime}(z)\right|$ for $|z| \geqq 1$. In particular $K^{n-1}\left|q^{\prime}\left(e^{i \theta}\right)\right| \leqq$ $K\left|p^{\prime}\left(K^{2} e^{i \theta}\right)\right|$ and the lemma follows.

Lemma 2. If $p(z)$ is a polynomial of degree $n$ having all its zeros in the disk $|z| \leqq K, K \geqq 1$, then

$$
\max _{|z|=1}\left|q^{\prime}(z)\right| \leqq K^{n} \max _{|z|=1}\left|p^{\prime}(z)\right|,
$$

where $q(z)$ is as defined in Lemma 1. 
Proof of Lemma 2. By Lemma 1,

$$
\max _{|z|=1}\left|q^{\prime}(z)\right| \leqq \frac{1}{K^{n-2}} \max _{|z|=K^{2}}\left|p^{\prime}(z)\right| .
$$

But if $f(z)$ is a polynomial of degree $n$ such that $|f(z)| \leqq M$ on $|z|=1$ then $|f(z)| \leqq M R^{n}$ for $|z|=R>1$ (see [3, Problem 269, page 137]). Hence $\max _{|z|=K^{2}}\left|p^{\prime}(z)\right| \leqq K^{2 n-2} \max _{|z|=1}\left|p^{\prime}(z)\right|$, from which the lemma follows.

LEMMA 3. If $p(z)=\sum_{v=0}^{n} a_{v} z^{v}$ is a polynomial of degree $n$,

$$
\max _{|z|=1}|p(z)|=1 \text {, }
$$

then on $|z|=1$

$$
\left|p^{\prime}(z)\right|+\left|q^{\prime}(z)\right| \leqq n,
$$

where $q(z)$ is as defined in Lemma 1.

This is a special case of a result due to Govil and Rahman [1, Lemma $10]$.

LEMMA 4. If $p(z)=\sum_{v=0}^{n} a_{v} z^{v}$ is a polynomial of degree $n$,

$$
\max _{|z|=1}|p(z)|=1, \quad p(z) \equiv q(z)
$$

where $q(z)$ is as defined in Lemma 1, then on $|z|=1$,

$$
\max _{|z|=1}\left|p^{\prime}(z)\right|=\frac{n}{2} \text {. }
$$

Proof OF Lemma 4. If $p(z) \equiv q(z)$, it follows by Lemma 3 ,

$$
\max _{|z|=1}\left|p^{\prime}(z)\right|=\max _{|z|=1}\left|q^{\prime}(z)\right| \leqq \frac{n}{2} .
$$

Since on $|z|=1,\left|q^{\prime}(z)\right|=\left|n p(z)-z p^{\prime}(z)\right|$ we get for $|z|=1$,

$$
n / 2 \geqq\left|q^{\prime}(z)\right|=\left|n p(z)-z p^{\prime}(z)\right| \geqq n|p(z)|-\left|p^{\prime}(z)\right| .
$$

Choosing $z$ on $|z|=1$ for which $|p(z)|$ becomes maximum, we get $\max _{|z|=1}\left|p^{\prime}(z)\right| \geqq n / 2$ and the lemma follows.

3. Proof of the theorem. For every $\varepsilon,|\varepsilon|=1$, the polynomial $P^{*}(z)=\frac{1}{2}\{p(z)+\varepsilon q(z)\}$ satisfies $P^{*}(z) \equiv z^{n}\left(P^{*}(1 / \bar{z})\right)^{-}$and $\max _{|z|=1}\left|P^{*}(z)\right|=$ 1 , hence by Lemma 4

$$
\max _{|z|=1}\left|p^{\prime}(z)+\varepsilon q^{\prime}(z)\right| \geqq n,
$$


which implies

$$
\max _{|z|=1}\left|p^{\prime}(z)\right|+\max _{|z|=1}\left|q^{\prime}(z)\right| \geqq n .
$$

Applying Lemma 2, we get

$$
\max _{|z|=1}\left|p^{\prime}(z)\right|+K^{n} \max _{|z|=1}\left|p^{\prime}(z)\right| \geqq n,
$$

and the theorem follows.

I am extremely grateful to Professor Q. I. Rahman for his kind help in the preparation of this paper.

\section{REFERENCES}

1. N. K. Govil and Q. I. Rahman, Functions of exponential type not vanishing in a half-plane and related polynomials, Trans. Amer. Math. Soc. 137 (1969), 501-517. MR 38 \#4681.

2. M. A. Malik, On the derivative of a polynomial, J. London Math. Soc. (2) 1 (1969), 57-60. MR 40 \#2827.

3. G. Pólya and G. Szegö, Aufgaben und Lehrsätze aus der Analysis. Vol. 1, Berlin, 1925.

4. P. Turán, Ueber die Ableitung von Polynomen, Compositio Math. 7 (1939), 89-95. MR 1, 37.

Department of Mathematics, Indian Institute of Technology, New Delhi-29, INDIA

Département de Mathématique, Université de Montréal, Montréal, Québec, CANADA 